\title{
Technology as applied to patient safety: an overview
}

\section{Rajesh Aggarwal, ${ }^{1}$ Oliver T Mytton, ${ }^{2}$ Felix Greaves, ${ }^{3}$ Charles Vincent ${ }^{1}$}

\section{INTRODUCTION}

Patient safety is a tough problem: tough in cultural, technical, clinical and psychological terms and because of its massive scale and heterogeneity. ${ }^{1}$ Healthcare encompasses many diverse areas: the mostly routine, but sometimes highly unpredictable and hazardous world of surgery, primary care and rehabilitation where patients have established relationships with their doctors over many years, the nature of probability in preventive medicine, some highly organised and ultrasafe processes such as the management of blood products and the inherently unpredictable, constantly changing environment of emergency medicine. In all these areas, error and harm to patients are real possibilities and frequent actualities, although the nature of the harm, its causes, consequences and likely methods of prevention will differ widely according to context. ${ }^{2}$

It is important to note that an increasing awareness of medical injuries has been paralleled by the rise in technology, and the increasing complexity it causes. The number of different medications for blood pressure, the various approaches and techniques for hip surgery and the multitude of blood tests for diagnosis of infections, all provide infinitely more opportunities for things to go wrong. This is not to say that the patient safety problem is caused by technology, but rather that technology has led to a complex and confusing environment within medicine that requires support systems in order for human beings to provide the correct treatments.

Many industries have made significant advances in safety through the adoption

\footnotetext{
${ }^{1}$ Division of Surgery, Department of Surgery \& Cancer, Imperial College London, UK; ${ }^{2}$ WHO Patient Safety, Department of Health, London, UK; ${ }^{3}$ Department of Health, Richmond House, London, UK

Correspondence to Mr Rajesh Aggarwal, Division of Surgery, Department of Surgery and Cancer, St Mary's Campus, Imperial College Healthcare NHS Trust, 10th Floor, Queen Elizabeth the Queen Mother Building, St Mary's Hospital, Praed Street, London W2 1NY, UK; rajesh.aggarwal@imperial.ac.uk
}

of technology. ${ }^{3} \quad$ Manufacturing has engaged in the reduction of human personnel through deployment of machine-based systems to produce highquality goods. In aviation, there has been explicit acknowledgement of the potential for human error and a conscious effort to reduce error through the considered use of technology and automation. ${ }^{4}$ However, these examples are quite different. The aviation paradigm has been translated to medicine, through the use of simulation, briefings, checklists and team working. All of the above are enablers of the use of technology to enhance workflow, organisation and 'getting the job done,' rather than sole implementation of a machine to 'do the job' of a human being.

Every clinical process is supported by numerous other systems and processes, variously dependent on technology. These include specific areas within the hospital environment, such as the role of pharmacies and laboratories in the generation and prevention of errors. The extent to which strict procedures and protocols are feasible or desirable in all facets of healthcare remains to be defined. Some healthcare processes, such as the delivery of medication, are analogous to production lines, and automated robotic systems have enormous potential in terms of efficiency of care, cost reduction and minimisation of error. ${ }^{5}$ Nonetheless, even these processes necessitate adequate staff and user-training prior to implementation. However, healthcare is a much more 'hands on' activity than many other high-risk industries. Healthcare requires skilled interventions, adaptation and flexibility and, most importantly, caring and empathy. Technology can support, but cannot replace human beings. It is crucial that existing as well as new technologies in terms of accuracy, efficiency and standardisation of care processes are not seen to undermine the quality and nature of the care received by patients.

Technology could improve safety and have other benefits, such as increased efficiency, but it can also present new hazards. ${ }^{6} 7$ The potential impact of technology, for both good and ill, will vary enormously depending on the setting and the tools and infrastructure in place. Systems such as electronic medical records could have great benefits in a metropolitan teaching hospital but be wholly inappropriate in low-resource settings where simpler systems would have a much greater effect.

This paper introduces a series of four reports examining the role that technology might play in enhancing the safety of healthcare, all derived from the WHO Patient Safety Technology programme. The paper sets out a systematic approach to identifying the potential role of technology and its impact on the clinical process and patient safety.

\section{WHO PATIENT SAFETY TECHNOLOGY PROGRAMME}

WHO Patient Safety has a longstanding interest in what technology can do to improve the safety of patient care. ${ }^{8}$ Aspects of technology, both low- and high-tech, have featured in its work: from the use of a simple alcohol hand rub to minimise risk of healthcare-associated infection, to a proposal to produce low-cost pulse oximeters. This series of papers marks the start of a more extensive programme of work on technology and patient safety designed to bring together these various threads and attempt to weave a cohesive narrative that identifies the role of technology in providing safer care. As part of this work, WHO Patient Safety set up four working groups to consider various aspects of the technology and patient safety interface. Two of the groups looked at cross-cutting themes, introducing new technology safely and making existing technologies safer-whereas the other two looked at specific areas-simulation and information technology (box 1).

The intention was to examine some, but not all, aspects of healthcare technology with particular reference to patient safety. The reports that follow, from each of the four working groups, have been derived from a variety of sources: expert consensus, literature review, published reports and experiential findings. The four working groups were multiprofessional and multidisciplinary in nature, in order to attempt to capture perspectives from around the globe. The four groups were each charged with a different aspect of technology and requested to focus upon current and future potential to enhance patient safety. Each group was also asked to take into account their role in improving the quality and efficiency of healthcare. 


\section{Box 1 WHO technology for patient safety programme}

An overview of the four working groups, with respect to the briefs for manuscript preparation.

\section{Introducing new technology safely}

To consider the role of regulation, health technology assessment, clinical engineering and training and surveillance in the introduction of new technologies. Particular consideration is given to the needs and challenges in developing-world settings.

\section{Making existing technologies safer}

To consider reporting systems, human factors, home care, hospital care, primary care and quality assurance. Particular consideration is given to the needs and challenges in developing world settings.

\section{Information technology}

To consider interventions like electronic records, computerised physician order entry (CPOE), decision support and bar coding. The importance of IT for data collection systems to facilitate reporting and learning is also emphasised. Some of the barriers to the wider implementation of IT, work-arounds, cost and institutional resistance are considered.

\section{Simulation and training}

To consider the potential of simulation to improve patient safety through education and training, moving the learning curve from patient to simulator. The use of both low and hightech simulation will be considered, as well as the potential for using simulation in research, design and testing.

The themes that quickly emerged were the paucity of literature from low-resource settings, a 'gap' between theoretical and empirical benefits of technology and the need for research to drive implementation of best practice. The potential of technology to cause harm was also discussed, together with the need to avoid a 'one size fits all' mentality. While this analysis related to the technology, there was also consideration of the infrastructure in terms of appropriate use, initial operatortraining and timely maintenance. The importance of information capture with respect to patient safety events and identification of harm was a crucial area where technological assistance could be vital.

\section{Case for medical technologies}

Technology is a broad concept, defined as 'the practical application of knowledge especially in a particular area,' and also as 'a capability given by the practical application of knowledge. ${ }^{9}$ Although medical technology is traditionally defined as manufactured devices to diagnose, treat and monitor disease processes, a wider application is possible. It can include sterile processes, delivery of anaesthesia, stock provision and radio-therapeutic interventions.

This report takes a broad definition of technology that encompasses both the tools and crafts used to deliver healthcare. In practice, the emphasis is on technologies with an explicit clinical application, both diagnostic and therapeutic, including devices, software, equipment and procedures. Supporting technologies, such as a generator to power a hospital, are given less consideration. Safety is a broad concept, but the intention is to focus on the impact of technology on patient safety, rather than occupational or environmental safety.

In order to consider the areas of technology and patient safety in a systematic manner, it was first necessary to provide a framework upon which to hang our thoughts. With this in mind, clinical processes were divided into four broad areas, with the effect of different technologies discussed in each context. The four clinical divisions were diagnostic, therapeutic, preventive and information-based domains. It is of course possible that the same technology could be used across these areas; for example, ultrasound might be diagnostic or may guide a therapeutic intervention. Examples of each broad area are provided in box 2 .

Various categorisations of medical technologies have been proposed that consider the utility of technology. When exploring the safety of technology in a global context there are two dimensions of interest (figure 1). First, the complexity of technology, and second, the spectrum from high-end to low-end technology. Complexity of the technology is often linked to cost, which is an important driver in both low- and high-resource settings. Complexity is also important in

\section{Box 2 Four clinical divisions used as a framework for considering new} technologies

\section{Diagnostic}

The advent of radiography and now CT, MRI and functional imaging allows unprecedented diagnostic access in ways that were unthinkable two decades ago.

\section{Therapeutic}

The use of minimally invasive therapies to treat disease has radically altered the patient experience for common procedures such as gallbladder removal and tubal sterilisation. This technology improved safety in many respects but also introduced new hazards; in some centres, bile duct and aortic injuries increased initially owing to the absence of adequate preparation, training and assessment prior to the routine use of the new techniques. $^{10}$

\section{Preventive}

The past two decades have witnessed widespread increases in screening practices for malignant and infectious diseases; these approaches offer the potential to reduce the burden of disease worldwide.

\section{Information-based}

Information technology (IT) is particularly critical to patient safety and the reduction of human error. ${ }^{11}$ It is the basis whereby information is provided to the healthcare worker through an automated system. In the past 50 years, there has been an explosion of medical knowledge. The sheer quantity of medical information, even within a single specialty, is often beyond the power of one person to comprehend. There is a sense in which modern medicine is a humanly impossible task; the human brain simply cannot cope with the volume and complexity of information needed to ensure patient safety. For instance, more than 600 drugs require adjustment of doses for multiple levels of renal dysfunction - an easy task for a computer but one that will inevitably be performed poorly by a person. 


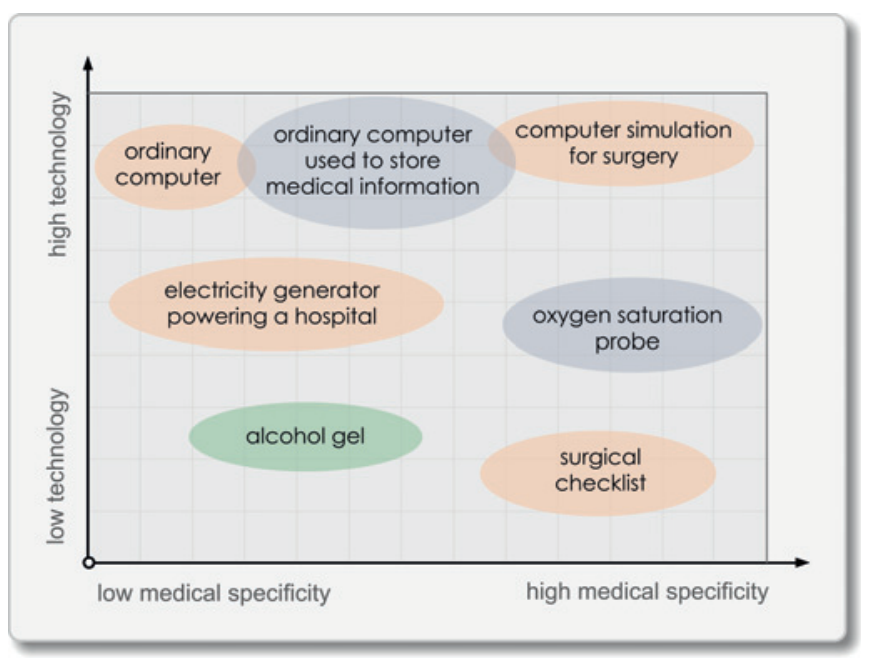

Figure 1 Illustration of the breadth and depth of medical technologies. The chart describes on the $x$-axis the degree of specialisation of a medical technology, and on the $y$-axis the level of complexity of the technology.

terms of ease of use, necessary training, need for regulation and degree of maintenance required. The second dimension of interest is the measure to which technology is medically specific. There is a natural and appropriate tendency to focus interest on technologies that are specific to medicine; however, the role of supporting technologies can be paramount in providing safe care. If the water supply to a hospital is limited, without backup, sterilisation practices could be compromised, with significant implications for patient safety.

Technology is clearly about more than high-end technologies, and its reach can be truly global. The nature of technology solutions must be tailored to local needs: environmental, ethical, cultural, social and economic. This is often termed 'appropriate technology.' Some of the WHO Patient Safety's key technology solutions fall into this category. ${ }^{12}$

It is also important to recognise that technologies can pose many hazards. Some of these relate to improper or careless use; for instance, if a technology is introduced too quickly and without thought for the culture and clinical setting or without appropriate training, it can become a danger rather than a benefit. Technologies pose hazards because of poor interface design or inadequate integration into existing systems, or because they slow clinical work to the point of inefficiency. A further point is the importance of proper servicing, maintenance of equipment and use of calibration tools to provide safe patient care. More subtly, a technology can bring enormous benefits in terms of the reduction of some kinds of errors but increase others. For instance, a computerised prescribing system can reduce dangerous drug interactions, but increase substitution errors of drugs with similar names because of the use of drop-down alphabetical menus (eg, prescribing dexamphetamine instead of dexamethasone). ${ }^{6}$

\section{Framework for analysis and action}

What role do drugs play in the treatment of disease? Immediately one sees that this question cannot be answered in its present form; one needs to know which type of disease, what kind of patient and which drug? Similarly, the question "what role can technology play in patient safety?' cannot be answered in this form. The question must be 'What role can technologies play in a particular setting with reference to a particular process?'

Framing the question in this way makes it clear that the first step must be to examine the clinical setting, current technology and the infrastructure in order to make a sensible assessment of the potential role of technology to enhance patient safety. To illustrate this approach, two examples of patient pathways have been examined: the first describes an emergency medical admission (figure 2) and the second an elective surgical admission (figure 3). These examples obviously do not encompass the entirety of healthcare, but they do cover some of the major procedures in all settings and also reflect some of the variety of provision and setting. The intention is for this framework to be transferable to other clinical processes, environments and resource settings.
We have pursued a pragmatic approach to considering the role of technology in supporting delivery of care by enacting real patient journeys in a variety of settings. The analysis proceeds in three stages:

- mapping the basic clinical process and technologies;

- examining potential errors, failures and vulnerabilities of the particular process;

- the role of technology in enhancing safety in the particular setting.

\section{Example 1: emergency medical care on} admission - acute chest pain (figure 2)

On arrival in an emergency department, the key initial technologies will be a medical record, oxygen saturation and blood pressure measuring devices, an electrocardiogram (ECG), and hospital pathways of care for acute chest pain.

Medical error at this stage can relate to diagnosis (eg, failure to interpret the ECG correctly), therapy (eg, thrombolysis with a background of recent haemorrhagic stroke, where primary angioplasty would be the preferred option), prevention (eg, missing a patient's antiepileptic drugs at admission leading to a seizure and injury), or information management (eg, receipt of the result of a raised Troponin I level).

Error in diagnosis, therapeutic intervention, prevention and information management are reducible. Technologies that can reduce such error include a single electronic record shared between primary and secondary care and decision support systems for ECG interpretation. Pathways, guidelines and mnemonics like MONA (morphine, oxygen, nitrate and aspirin for acute cardiac chest pain) can help to standardise care and drive high quality and safety in provision of care. A classic example hails from acute life support, which is a standardised method of performing a life-saving procedure for the emergency team. Simulated scenarios can enable healthcare professionals to put their knowledge into practice, and provide accurate feedback through observation of their performance. ${ }^{13}$

\section{Example 2: elective surgical care in the operating room-laparoscopic cholecystectomy (figure 3)}

A patient undergoing an invasive procedure in the operating room is primarily subject to anaesthetic and surgical technologies. The airway, breathing and circulation are under constant monitoring from electronic devices, providing the anaesthetist with a dynamic physiological 'report card' of their patient. Nonetheless, this is dependent upon a clear 


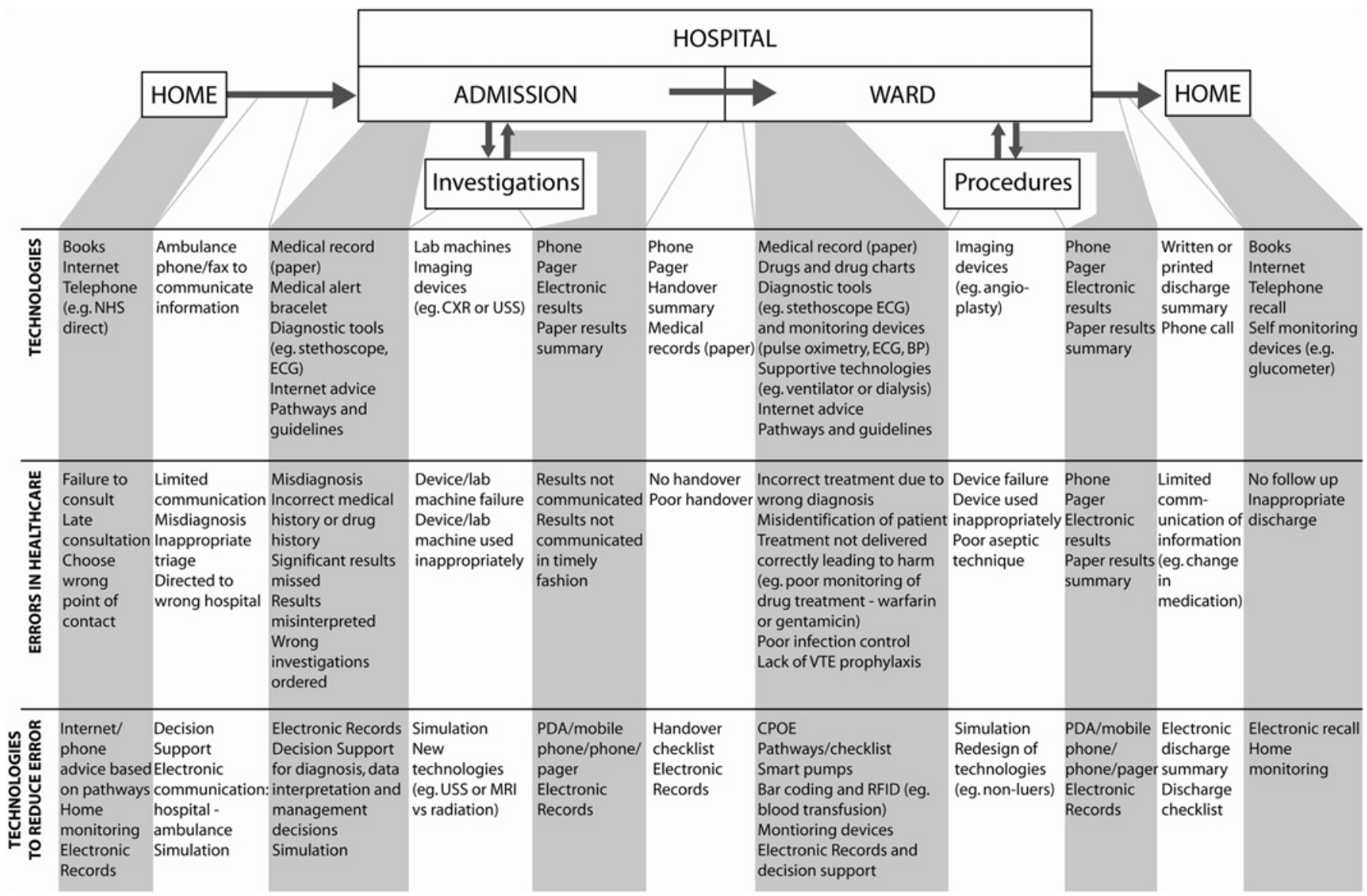

Figure 2 Emergency medical care on admission-acute chest pain. The flow pathway is for a patient with acute chest pain, from hospital admission to discharge. At each stage of the pathway, technologies currently in use, potential healthcare errors and technologies to reduce error are defined.

understanding of the information provided to the anaesthetist. The surgeon is operating with specialised tools that could include high-energy devices such as diathermy and radiofrequency waves.

Medical error can relate to diagnosis (eg, failure to interpret the surgical anatomy correctly), therapy (eg, intraoperative damage to adjacent tissues leading to bleeding), prevention (eg, failure to administer prophylactic antibiotics) or information management (eg, loss of preoperative endoscopic retrograde cholangio-pancreatogram (ERCP) images leading to an unnecessary intraoperative cholangiogram).

In analogy to the medical pathway for acute chest pain, surgical error is reducible for diagnosis, therapeutic intervention, prevention and result interpretation. Technologies that could reduce such error include intraoperative guidance, through the use of infrared cameras to delineate biliary structures, and protocol-driven processes within the operating room to ensure administration of appropriate medication (eg, antibiotics, insulin and flowtron boots). Particularly within the surgical technical domain, virtual reality simulation has been shown to shorten and flatten the learning curve for laparoscopic procedures in the operating room, with a reduction in time and error and concomitant enhancement in operator dexterity. $^{14}$

\section{Emerging themes and wider systems}

We believe the patient pathway provides a useful method to consider the relationship between technology and patient safety. The intention is to provide a view to designing and introducing technologies to reduce error. The great power of this approach is that it is structured and simple with the ability to be specified to a particular process, setting, environment or cultural domain. Through the illustrative analyses, a number of initial themes have emerged:

- the importance of improved communication between domains of care;

- the value of pathways, checklists and guidelines to standardise care;

- the use of Computerised Physician Order Entry (CPOE) systems to help address medication administration errors;

- the use of electronic records to support decision-making and promote the exchange of information;
- the value of training outside the clinical environment, in an educational setting. Although beginning with the patient pathway ensures relevance and applicability to that clinical setting, it is only a starting-point in examining the role of technology.

Wider factors surrounding a patient, at local, national and international level, and how technology effects these also needs to be considered. Significantly, at local and national levels, IT systems for reporting and learning, when properly applied, have a vital role in reducing error. Wider issues, such as the role of regulation and standards for the safe introduction of both new and existing technologies, are not explicitly considered in this pathway.

\section{DISCUSSION}

Recent times have witnessed an explosion in the development and implementation of healthcare technologies. This has been paralleled by increases in healthcare expenditure, especially within highresource regions. Associative factors of an ageing population, chronic diseases, sophisticated prescription medication and 


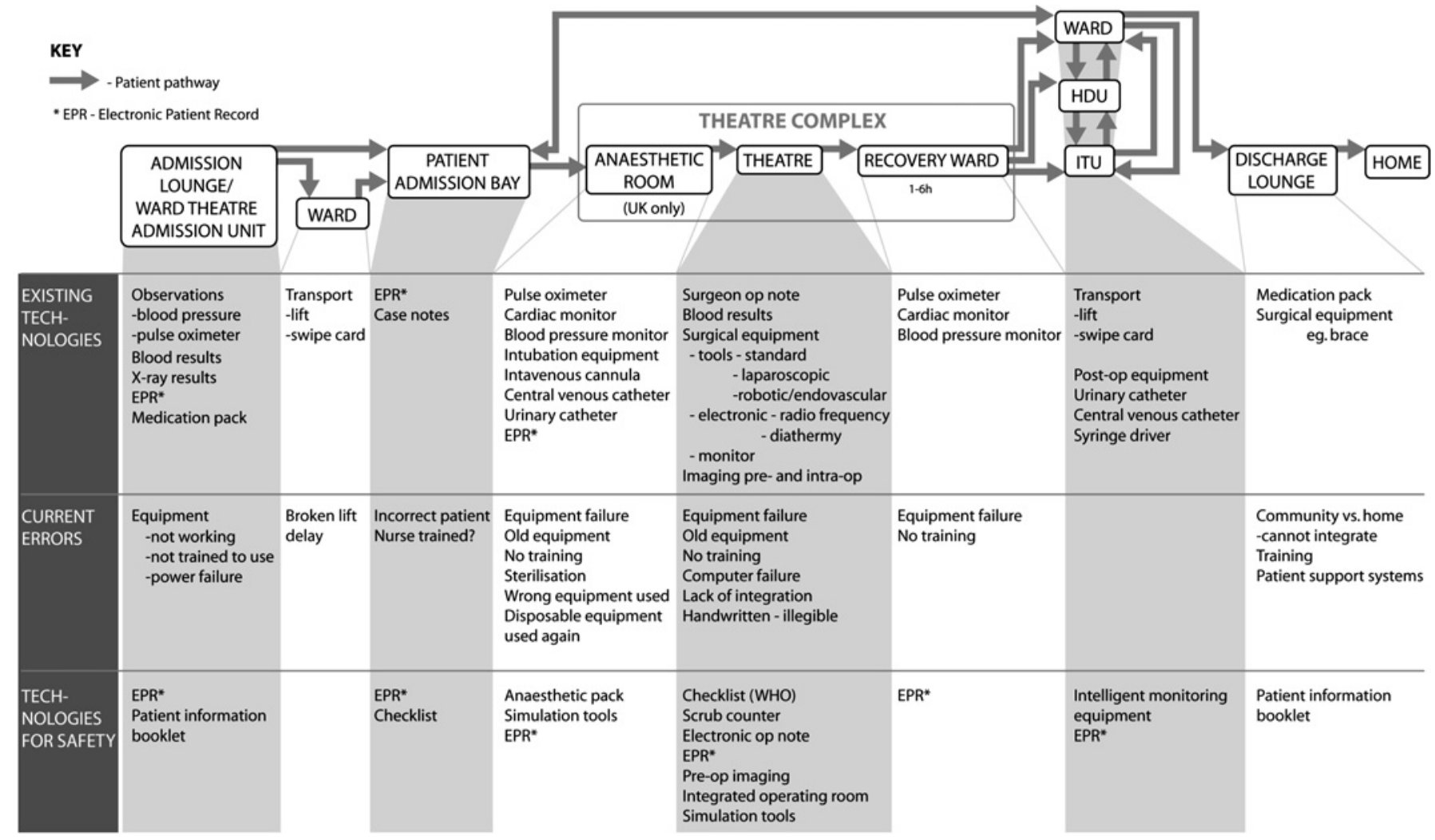

Figure 3 Elective surgical care in the operating room-laparoscopic cholecystectomy. The flow pathway is for a patient to undergo laparoscopic surgery, from hospital admission through to discharge. At each stage of the pathway, technologies currently in use, potential healthcare errors and technologies to reduce error are defined.

advanced intervention technologies have fuelled extensive debate with regards to the future management of health services. Within the midst of this melee are reports that one in 10 patients suffers harm during their medical journey. Nonetheless, there is now a concerted effort to enhance levels of patient safety. Patient safety is a recognised part of modern medicine, as witnessed by the development of national patient safety agencies and initiatives that have the potential to influence national and international health policy. The WHO Patient Safety programme aims to coordinate, disseminate and accelerate improvements in patient safety worldwide. One part of the programme was to investigate the role of technology with regard to patient safety.

Within the realm of technology and its application to safety, there is often reference to other high-reliability organisations such as nuclear, oil and the military. These industries use high-end technologies to prevent, diagnose and treat error. However, integral to their approach is the need to involve the end user at appropriate points within their product pathway. The commonly cited Crew Resource Management (CRM) literature stems from the aviation industry and has enhanced safety through the creation of a culture of safe practice with nothing left to chance. Recently, there have been proposals to translate such gains into medicine, including the development of checklists, enhanced communication, the use of simulation, the importance of debriefing and redesign of connector parts. ${ }^{15} 16$

Medicine is an extremely disparate faculty, however, ranging from routine processes of care in a hospital ward, through to mass casualties in a field environment. The application of technology to enhance patient safety must thus be considered with respect to the clinical process, currently employed technologies, appropriate equipment maintenance and the environment of care. It is against this background that this overview paper has developed a simple, structured and generic framework for consideration of the relationship between technology and patient safety. The intention is that this framework could be applied to a number of other clinical pathways, in a variety of settings. With this approach, it may be possible not only to enhance such clinical pathways but also to provide an overview of where technology can have its biggest gains with regards to patient safety. For example, the role of handover is extremely important across a number of clinical pathways, and as such can be demonstrated and applied appropriately. The pathway approach can also illustrate the transference of error across clinical areas, for example, from ward to operating theatre.

The findings outlined in the following four manuscripts have sought to provide a snapshot of the current status and future possibilities with respect to technology and patient safety. The articles are intended not to provide an exhaustive review of the literature but rather to provide a starting-point for future empirical work. It is though with excitement that we proceed along this journey in order to enhance patient safety in the digital age. The final thought perhaps is that the greatest gains are likely to come from simple interventions that are applicable to a significant proportion of our patients. Finding them is the challenge.

Funding The project was funded by WHO Patient Safety.

\section{Competing interests None.}

Provenance and peer review Not commissioned; externally peer reviewed.

Qual Saf Health Care 2010;19:i3-i8 doi:10.1136/qshc.2010.040501 


\section{REFERENCES}

1. Vincent C, Taylor-Adams S, Stanhope N. Framework for analysing risk and safety in clinical medicine. BMJ 1998:316:1154-7.

2. Kohn Linda T, Corrigan Janet M, Donaldson Molla S. To err is human: building a safer health system. Washington: National Academy Press, 2000.

3. Hetherington C, Flin R, Mearns K. Safety in shipping: the human element. J Safety Res 2006;37:401-11.

4. Pronovost PJ, Goeschel CA, Olsen KL, et al. Reducing health care hazards: lessons from the commercial aviation safety team. Health Aff (Millwood) 2009; 28:w479-89.

5. Cytocare. CytoCare Robot [Internet communication], 2009. http://www.cytocare.info/.

6. de Wildt SN, Verzijden R, van den Anker JN. de HM Information technology cannot guarantee patient safety. BMJ 2007;334:851-2.
7. Murphy DG, Bjartell A, Ficarra V, et al. Downsides of robot-assisted laparoscopic radical prostatectomy: limitations and complications. Eur Urol. Published Online First: 25 December 2009.

8. Donaldson LJ, Fletcher MG. The WHO world alliance for patient safety: towards the years of living less dangerously. Med J Aust 2006;184(Suppl 10): S69-72.

9. Webster's Third New International Dictionary, Unabridged. Springfield, MA: Merriam-Webster Inc, 2009.

10. Apelgren KN, Scheeres DE. Aortic injury. A catastrophic complication of laparoscopic cholecystectomy. Surg Endosc 1994;8:689-91.

11. Bates DW, Gawande AA. Improving safety with information technology. N Engl J Med 2003; 348:2526-34.

12. Pittet D, Allegranzi B, Storr J, et al. 'Clean care is safer care': the global patient safety challenge
2005-2006. Int J Infect Dis 2006

10:419-24.

13. Wayne DB, Didwania A, Feinglass J, et al Simulation-based education improves quality of care during cardiac arrest team responses at an academic teaching hospital: a case-control study. Chest 2008;133:56-61.

14. Aggarwal R, Ward J, Balasundaram I, et al. Proving the effectiveness of virtual reality simulation for laparoscopic surgical training. Ann Surg 2007;246:771-9.

15. Haynes AB, Weiser TG, Berry WR, et al. A surgical safety checklist to reduce morbidity and mortality in a global population. N Engl J Med 2009; 360:491-9.

16. Bates DW, Leape LL, Cullen DJ, et al. Effect of computerized physician order entry and a team intervention on prevention of serious medication errors. JAMA 1998;280:1311-16 